In this paper, I will provide a brief history of Whooping Cough and its associated pathogens, B. pertussis and B. parapertusis. I will then address some modern concerns, due to recent outbreaks and new research bringing into question the efficacy and effectiveness of existing vaccines. Finally, I will suggest additional concerns and in some cases, provide a few suggestions for research proposals which can address these concerns. This work is partially an integration of less formal discussions on the topic that I have written on my personal blogs, including one that constitutes a very early draft of this paper ${ }^{1}$.

Whooping cough is a disease, which is primarily caused by the gram-negative B. pertussis. however a milder form of symptoms can be caused by the closely related B. parapertussis ${ }^{2}$. The strains of these pathogens seem to be fairly host specific ${ }^{3}$. B. pertussis was first isolated in $1906^{4}$. According to CDC data, from 1922 through 1947, there were over 100,000 cases of Whooping Cough per year. Since then, cases have dropped significantly. In 1976, there were only 1,010 cases.

However, over the last few decades, cases of Whooping Cough have been on the rise, with over 48,277 cases in 2012..$^{5}$ Vaccines likely had a major role to play in the decline of case reports. The first vaccines were being developed by the early 20 s and whole cell vaccines were developed in the $30 \mathrm{~s}^{6}$. However, due to perceived risks of the whole cell vaccine, acellular vaccines were developed in the $80 \mathrm{~s}^{7}$. Because of the risks associated Whooping Cough, a better understanding of why we are seeing a resurgence, identifying how many people are infected with the B. pertussis and B. parapertussis, and modeling the implications of these results are all crucial for protecting the population.

A number of explanations have been proposed, for the recent uptick in incidence rates. In the media, one of the suggested causes being discussed is under-vaccination ${ }^{8}$. However a line of

\footnotetext{
${ }^{1}$ Daniel Goldman, "On the Scientific Perception and Reality of Whooping Cough," The Spiritual Anthropologist, April 05, 2018, , accessed June 28, 2018, http://spiritualanthropologist.info/new-research/on-the-scientific-perception-and-reality-of-whooping-cough/. ${ }^{2}$ Elahi, Shokrollah, David R. Thompson, Stacy Strom, Brendan O'Connor, Lorne A. Babiuk, and Volker Gerdts. "Infection With Bordetella Parapertussis but Not Bordetella Pertussis Causes Pertussis-Like Disease in Older Pigs." The Journal of Infectious Diseases 198, no. 3 (2008): 384-92. doi:10.1086/589713.

${ }^{3}$ Ibid.

${ }^{4}$ Cherry, James D. "The History of Pertussis (Whooping Cough); 1906-2015: Facts, Myths, and Misconceptions." Current Epidemiology Reports 2, no. 2 (2015): 120-30. doi:10.1007/s40471-015-0041-9.

5 "Pertussis (Whooping Cough)." Centers for Disease Control and Prevention. February 07, 2018. Accessed June 17, 2018. https://www.cdc.gov/pertussis/surv-reporting/cases-by-year.html.

${ }^{6}$ Cherry, James D. "The History of Pertussis (Whooping Cough); 1906-2015: Facts, Myths, and Misconceptions." Current Epidemiology Reports 2, no. 2 (2015): 120-30. doi:10.1007/s40471-015-0041-9.

${ }^{7}$ Ibid.

8 "Vaccine Refusal Leads to Rise in Measles \& Whooping Cough." Healthline. Accessed June 17, 2018. https://www.healthline.com/health-news/measles-whooping-cough-rise-driven-by-unvaccinated-people.
} 
evidence suggests another cause. First, in the last decade or so, it has come to the attention of researchers that the acellular B. pertussis vaccines might only offer limited ability to prevent the colonization and transmission of the pathogen ${ }^{9}$. Unfortunately, even returning to the whole cell vaccine may not be sufficient to prevent the rise in $\operatorname{cases}^{10}$.

One of the potential causes of recent increases in cases is a mismatch between the vaccine strains and circulating strains ${ }^{11}$. Additionally, it has been suggested that Whooping Cough vaccines, including the acellular vaccines, wane in their efficacy over time ${ }^{12}$. Unfortunately, simply adding more doses of the vaccine seems to be insufficient, as even after a fifth dose, immunity wanes so rapidly that one year after the final dose, the odds of acquiring the infection can increase by $42 \%$ 13.

\section{Concerns and Proposals}

Because of this information, there are a number of concerns about the spread of B. pertussis and related pathogens, as well as any pathogen which responds to a vaccine in a similar fashion. The following proposals will be made in this section. First, based on past research, a study investigating the rate of asymptomatic B. pertussis and B. parapertussis infections, within the United States, should be conducted. Additionally, an updated SIR-like model should be developed, in order to take into account how asymptomatic infections influence risk aversion and the spread of pathogens. Finally, a systematic review of available research on Whooping Cough vaccines should be conducted, to see what data is available regarding their efficacy, and whether or not studies are conflating ability to prevent infection and ability to prevent disease. This study should be coupled with a survey asking questions about the perception of current Whooping Cough vaccines.

\footnotetext{
${ }^{9}$ Warfel, J. M., L. I. Zimmerman, and T. J. Merkel. "Acellular pertussis vaccines protect against disease but fail to prevent infection and transmission in a nonhuman primate model." Proceedings of the National Academy of Sciences 111, no. 2 (2013): 787-92. doi:10.1073/pnas.1314688110.

${ }^{10}$ Srugo, Isaac et al. "Pertussis Infection in Fully Vaccinated Children in Day-Care Centers, Israel." Emerging Infectious Diseases 6, no. 5 (2000): 526-29. doi:10.3201/eid0605.000512.

${ }^{11}$ Hester De Melker, "Reemergence of Pertussis in the Highly Vaccinated Population of The Netherlands: Observations on Surveillance Data," Emerging Infectious Diseases 6, no. 4 (2000): , doi:10.3201/eid0604.000404.

${ }^{12}$ Nicola P. Klein et al., "Waning Protection after Fifth Dose of Acellular Pertussis Vaccine in Children," New England Journal of Medicine 367, no. 11 (2012): , doi:10.1056/nejmoa1200850.

${ }^{13}$ Ibid.
} 
In 2014, a group of researchers set out to investigate the prevalence of asymptomatic B. pertussis and B. parapertussis infections, among school aged children in China. Both PCR and culture methods were used. ${ }^{14}$

The results from the research on asymptomatic infections within Chinese school aged children opens up the possibility that the United States and other developed countries, with high vaccination rates, may still experience much higher than believed rates of infection. In order to approximate how many different populations in which the B. pertussis pathogen can evolve, a study similar to Zhang et al. 2014 should be conducted, but with a more general population, within the United States, and possibly other countries as well. In the original study, only 629 subjects were tested, and of those, only 2 were confirmed to be infected with B. pertussis, and 1 was confirmed to be infected with B. parapertussis, when using the culture test ${ }^{15}$.

Assuming that the rate of asymptomatic infections, in the United States, is at least 2 per 629 and the population is fairly homogeneous, then a sample of 1,447 should result in at least one confirmed infection, with a probability of $99 \%$. On the other hand, to obtain a $95 \%$ confidence level, with a relative standard error of 10 , assuming the true proportion is only 2 per 629 , the sample size would have to be nearly 50,000. Such a large sample is likely to be cost prohibitive, so without a large budget, it may be sufficient to perform the analysis on a sample of about 1,500 .

With a larger budget, the study could be stratified. Because this paper is suggesting the theory that vaccination leads to differences in asymptomatic infection rates, it might benefit us to select samples from both subpopulations. Another potential strata could be state, as it would be useful to see if different states have different rates of infection. However that strata would require a much larger budget. Finally, while the sample size is designed to ensure that some information about culture confirmed infections is captured, PCR testing should also be done.

Aside from knowing how many infected individuals there are in the population, it is also necessary to model the spread of infection, in a way that takes into account asymptomatic carriers. One method of modeling the spread of infection is by developing compartmental models, such as the SIR or the slightly more complicated SEIR model, which takes into account a lag period between being infected and being infectious ${ }^{16}$. But these models do not take into account asymptomatic carriage. Researchers, such as Martin Grunnill, have done some research

\footnotetext{
${ }^{14}$ Qi Zhang et al., "Prevalence of Asymptomatic Bordetella Pertussis and Bordetella Parapertussis Infections among School Children in China as Determined by Pooled Real-time PCR: A Cross-sectional Study," Scandinavian Journal of Infectious Diseases 46, no. 4 (2014): , doi:10.3109/00365548.2013.878034.

${ }^{15}$ Ibid.

${ }^{16}$ Brauer, Fred. "Mathematical Epidemiology: Past, Present, and Future." Infectious Disease Modelling 2, no. 2 (2017): 113-27. doi:10.1016/j.idm.2017.02.001.
} 
into how asymptomatic infections impact transmission. However, Grunnill's work focuses on dengue, and does not take into account vaccination. ${ }^{17}$

A variable, which seems like it should affect transmission rates, is risk aversion. While there are a number of complicated features of pathogen transmission, as indicated in the various models that have been addressed in this paper, two overall factors are involved in the rate of transmission: the probability that an infected individual will come into contact with a susceptible individual, and the easy at which transmission occurs, when a contact happens. It is recognized that humans tend to avoid disease or appearance of disease ${ }^{18}$. Therefore, reduced symptoms, while beneficial for the health of the individual being vaccinated, could result in greater contact between individuals. Understanding risk aversion requires the inclusion of psychological theory and the estimation of real world avoidance rates, based on various symptoms. Once these parameters are estimated, an epidemiological model, taking into account symptomatic and asymptomatic vectors can be developed. This model can be compared with real world rates of cases, and with the data from the above suggested prevalence study, and can be used to predict how prevalent infections and cases can become.

Another concern is as follows. One of the factors affecting the rate of evolution is population $\operatorname{size}^{19}$. If a vaccine prevents clinical symptoms, that is of course important, but a failure to prevent colonization and transmission can lead to a significant reservoir in which the pathogen can evolve become more virulent, especially if a vaccine can affect the evolution of the pathogen. It is well understood that antibiotics affect bacterial evolution by changing selective pressure. The first successful antimicrobials were developed in the late 30s, and streptomycin was introduced to combat turberculosis in 1944. Soon after, it was discovered that some strains of TB were developing resistance to the antibiotic. ${ }^{20}$ So as antibiotics were introduced, selective pressures drove the evolution of bacteria towards antibiotic resistance. But while antibiotic resistance is common, the evolution of vaccine resistance is rare ${ }^{21}$.

\footnotetext{
${ }^{17}$ Grunnill, Martin. "An Exploration of the Role of Asymptomatic Infections in the Epidemiology of Dengue Viruses through Susceptible, Asymptomatic, Infected and Recovered (SAIR) Models." Journal of Theoretical Biology 439 (2018): 195-204. doi:10.1016/j.jtbi.2017.12.009.

${ }^{18}$ Kouznetsova, Daria, Richard J. Stevenson, Megan J. Oaten, and Trevor I. Case. "Disease-avoidant behaviour and its consequences." Psychology \& Health 27, no. 4 (2012): 491-506. doi:10.1080/08870446.2011.603424.

${ }^{19}$ Frean, M., P. B. Rainey, and A. Traulsen. "The Effect of Population Structure on the Rate of Evolution." Proceedings of the Royal Society B: Biological Sciences 280, no. 1762 (2013): 20130211. doi:10.1098/rspb.2013.0211.

${ }^{20}$ J. Davies and D. Davies, "Origins and Evolution of Antibiotic Resistance," Microbiology and Molecular Biology Reviews 74, no. 3 (2010): , doi:10.1128/mmbr.00016-10.

${ }^{21}$ David A. Kennedy and Andrew F. Read, "Why Does Drug Resistance Readily Evolve but Vaccine Resistance Does Not?" Proceedings of the Royal Society B: Biological Sciences 284, no. 1851 (2017): , doi:10.1098/rspb.2016.2562.
} 
While vaccine resistance may not be as common as antibiotic resistance, vaccines are thought to have a number of evolutionary impacts on virulence ${ }^{22}$. Research has suggested that vaccines can cause virulence to increase, if they reduce the natural harm caused by the pathogen ${ }^{23}$. In a way, while antibiotics impose a selective pressure, with advantage given to pathogens that happen to evolve resistance to the antibiotic, vaccines which reduce the symptoms of a pathogen remove a selective pressure against virulence. Furthermore, referring back the increase in population affected by the pathogen, which could occur because of a partially effective vaccine, there are more organisms and therefore more chances for evolution to give rise to resistance or increased virulence.

Finally, it is concerning just how long it has taken the medical community to identify issues with the current vaccines, and identify limited efficacy. Usually a drug needs to be tested and efficacy justified, before it is put into widespread use, and as mentioned earlier, the first Whooping Cough vaccine were produced in the 1920 s and 30s. Furthermore, two acellular vaccines have been in use since $1991^{24}$. But it wasn't until ca. 2013 that it was identified that the acellular vaccines prevent the disease, but not colonization or transmission ${ }^{25}$. This leads to the question, "how much do we really know about the efficacy of Whooping Cough vaccines and how does this knowledge compare to the perception of knowledge in the medical community?" Answering this question requires a two part study. The first part would involve a systematic review of the available literature.

The systematic review would look at the amount of available research on the current Whooping Cough vaccines, with respect to their ability to prevent clinical symptoms, as well as their ability to prevent colonization and transmission, both in clinical settings and real world settings. Studies should also be checked for quality, especially in terms of accidental conflation between ability to prevent disease and ability to prevent colonization and transmission. Because an asymptomatic infection, or even a subclinical infection, would likely fail to result in a hospital visit, measuring infection rates from hospital populations is likely to underestimate infections. Even a vaccine, which reduces symptoms but not infections, will appear effective at preventing the spread of infection, if cases and infections are conflated.

\footnotetext{
${ }^{22}$ Clayton E. Cressler et al., "The Adaptive Evolution of Virulence: A Review of Theoretical Predictions and Empirical Tests," Parasitology 143, no. 07 (2015): , doi:10.1017/s003118201500092x.

${ }^{23}$ Ibid.

24 "Pertussis Vaccination: Use of Acellular Pertussis Vaccines Among Infants and Young Children Recommendations of the Advisory Committee on Immunization Practices (ACIP)," Centers for Disease Control and Prevention, , accessed June 28, 2018, https://www.cdc.gov/mmwr/preview/mmwrhtml/00048610.htm.

${ }^{25}$ Warfel, J. M., L. I. Zimmerman, and T. J. Merkel. "Acellular pertussis vaccines protect against disease but fail to prevent infection and transmission in a nonhuman primate model." Proceedings of the National Academy of Sciences 111, no. 2 (2013): 787-92. doi:10.1073/pnas.1314688110.
} 
The second half of the study would be a survey of the medical community, including researchers and physicians. If there are available resources, non-professionals could also be surveyed to see how the perception of Whooping Cough vaccine effectiveness varies between the medical community and the average person. The survey would ask five main questions. First it would ask for a guess for the rate of asymptomatic infections within the United States. The survey would then ask how effective the current vaccines are at preventing Whooping Cough disease. Then it would ask for a guess on the quality of the available evidence justifying effectiveness. Then it would ask how effective current vaccines are at preventing the colonization and transmission of B. pertussis. And finally it would ask for a guess of the quality of medical research justifying that position as well.

We need to know how much we know, about the efficacy and effectiveness of Whooping Cough vaccines, and expand upon that knowledge as much as possible. The influence vaccines have on virulence and transmission rates need to be understood. Newer models are needed in order to identify how asymptomatic carriage, associated with partial efficacy of a vaccine, influences the spread of pathogens. These are some of the concerns associated with vaccines, especially Whooping Cough vaccines. So, while there is little doubt that vaccines are a powerful class of medicines and have saved lives by reducing symptoms, but we cannot only worry about the short term benefits of vaccines. The long term effects of vaccination have to be considered as well.

Finally, this paper has provided a number of already existing results, which bring into question whether the Whooping Cough vaccines, in use today, have any real ability to prevent the spread of infection. There is a political issue here. If the vaccines do not prevent infection, mandatory vaccination, while likely to help those who are vaccinated, would offer little to no protection for those who cannot be vaccinated. Additionally, if the spread of the B. pertussis infection is not due to under-vaccination, attacks on the so called anti-vax movement constitute little more than a witch hunt. Science, not politics, needs to drive medical protocols. Therefore this research needs to be conducted as quickly as possible. 


\section{Works Cited}

Brauer, Fred. "Mathematical Epidemiology: Past, Present, and Future." Infectious Disease Modelling 2, no. 2 (2017): 113-27. doi:10.1016/j.idm.2017.02.001.

Cherry, James D. "The History of Pertussis (Whooping Cough); 1906-2015: Facts, Myths, and Misconceptions." Current Epidemiology Reports 2, no. 2 (2015): 120-30. doi:10.1007/s40471-015-0041-9.

Cressler, Clayton E., David V. Mcleod, Carly Rozins, Josée Van Den Hoogen, and Troy Day. "The Adaptive Evolution of Virulence: A Review of Theoretical Predictions and Empirical Tests." Parasitology 143, no. 07 (2015): 915-30. doi:10.1017/s003118201500092x.

Davies, J., and D. Davies. "Origins and Evolution of Antibiotic Resistance." Microbiology and Molecular Biology Reviews 74, no. 3 (2010): 417-33. doi:10.1128/mmbr.00016-10.

Elahi, Shokrollah, David R. Thompson, Stacy Strom, Brendan O'Connor, Lorne A. Babiuk, and Volker Gerdts. "Infection With Bordetella Parapertussis but Not Bordetella Pertussis Causes Pertussis-Like Disease in Older Pigs." The Journal of Infectious Diseases 198, no. 3 (2008): 384-92. doi:10.1086/589713.

Frean, M., P. B. Rainey, and A. Traulsen. "The Effect of Population Structure on the Rate of Evolution." Proceedings of the Royal Society B: Biological Sciences 280, no. 1762 (2013): 20130211. doi:10.1098/rspb.2013.0211.

Goldman, Daniel. "On the Scientific Perception and Reality of Whooping Cough." The Spiritual Anthropologist. April 05, 2018. Accessed June 28, 2018.

http://spiritualanthropologist.info/new-research/on-the-scientific-perception-and-reality-of-whoo ping-cough/.

Grunnill, Martin. "An Exploration of the Role of Asymptomatic Infections in the Epidemiology of Dengue Viruses through Susceptible, Asymptomatic, Infected and Recovered (SAIR) Models." Journal of Theoretical Biology 439 (2018): 195-204. doi:10.1016/j.jtbi.2017.12.009.

Kennedy, David A., and Andrew F. Read. "Why Does Drug Resistance Readily Evolve but Vaccine Resistance Does Not?" Proceedings of the Royal Society B: Biological Sciences 284, no. 1851 (2017): 20162562. doi:10.1098/rspb.2016.2562. 
Klein, Nicola P., Joan Bartlett, Ali Rowhani-Rahbar, Bruce Fireman, and Roger Baxter. "Waning Protection after Fifth Dose of Acellular Pertussis Vaccine in Children." New England Journal of Medicine 367, no. 11 (2012): 1012-019. doi:10.1056/nejmoa1200850.

Kouznetsova, Daria, Richard J. Stevenson, Megan J. Oaten, and Trevor I. Case. "Disease-avoidant behaviour and its consequences." Psychology \& Health 27, no. 4 (2012): 491-506. doi:10.1080/08870446.2011.603424.

Melker, Hester De. "Reemergence of Pertussis in the Highly Vaccinated Population of The Netherlands: Observations on Surveillance Data." Emerging Infectious Diseases 6, no. 4 (2000): 348-57. doi:10.3201/eid0604.000404.

"Pertussis Vaccination: Use of Acellular Pertussis Vaccines Among Infants and Young Children Recommendations of the Advisory Committee on Immunization Practices (ACIP)." Centers for Disease Control and Prevention. Accessed June 28, 2018. https://www.cdc.gov/mmwr/preview/mmwrhtml/00048610.htm.

"Pertussis (Whooping Cough)." Centers for Disease Control and Prevention. February 07, 2018. Accessed June 17, 2018. https://www.cdc.gov/pertussis/surv-reporting/cases-by-year.html.

Srugo, Isaac, Daniel Benilevi, Ralph Madeb, Sara Shapiro, Tamy Shohat, Eli Somekh, Yossi Rimmar, Vladimir Gershtein, Rosa Gershtein, Esther Marva, and Nitza Lahat. "Pertussis Infection in Fully Vaccinated Children in Day-Care Centers, Israel." Emerging Infectious Diseases 6, no. 5 (2000): 526-29. doi:10.3201/eid0605.000512.

"Vaccine Refusal Leads to Rise in Measles \& Whooping Cough." Healthline. Accessed June 17, 2018.

https://www.healthline.com/health-news/measles-whooping-cough-rise-driven-by-unvaccinatedpeople.

Warfel, J. M., L. I. Zimmerman, and T. J. Merkel. "Acellular pertussis vaccines protect against disease but fail to prevent infection and transmission in a nonhuman primate model." Proceedings of the National Academy of Sciences 111, no. 2 (2013): 787-92. doi:10.1073/pnas.1314688110.

Zhang, Qi, Zundong Yin, Yixing Li, Huiming Luo, Zhujun Shao, Yuan Gao, Li Xu, Biao Kan, Shan Lu, Yan Zhang, Manshi Li, Meizhen Liu, Pingping Yao, Zhixian Zhao, and Qiushui He. "Prevalence of asymptomatic Bordetella pertussis and Bordetella parapertussis infections among school children in China as determined by pooled real-time PCR: A cross-sectional study." 
Scandinavian Journal of Infectious Diseases 46, no. 4 (2014): 280-87. doi:10.3109/00365548.2013.878034. 